\title{
Characteristics of Chua's circuit based on memristor and responses to stimulating signals
}

\author{
Yujie $\mathrm{CaO}^{1, \mathrm{a}}$, Shuai $\mathrm{Ye}^{2, \mathrm{~b}}$, Chunxiao $\mathrm{Hu}^{3, \mathrm{c}}$ and Zhikuo Tao ${ }^{4, \mathrm{~d}}$ \\ College of Electronic Science and Engineering, Nanjing University of Posts and \\ Telecommunications, Nanjing, China, 210046 \\ ab13020110@njupt.edu.cn, bb13020114@njupt.edu.cn, cb13020407@njupt.edu.cn, dzktao@njupt.e \\ du.cn
}

Keywords: memristor, Chua's circuit, stimulating signals, transition of states

\begin{abstract}
Memristor is the fourth fundamental circuit element predicted by Leon O. Chua theoretically in 1971 and realized by HP scientists in 2008. In this work, we investigate the characteristics of canonical Chua's circuit and Chua's circuit based on memristor. More importantly, the response characteristics of the circuits under the stimulating signals are researched. It is suggested that the external stimulating signals could change the circuit states significantly, and the transition is dependent on the parameters.
\end{abstract}

\section{Introduction}

In 1971, the memristor was predicted by Leon O. Chua theoretically based on symmetry arguments[1]. As we all know, there are four fundamental circuit variables: electric current $i$, voltage $u$, charge $q$ and magnetic flux $\varphi$, and they are connected by five different mathematical relations (i.e. $d v=R d i, d q=i d t, d \varphi=L d i, d q=C d v, d \varphi=v d t$ ). Although, there is still a missing link between charge $q$ and magnetic flux $\varphi$, and charge controlled memristor defined by Eq. 1 connected these two variables where $M$ is memristance of the memristor. Meanwhile, the flux controlled memristor is defined by Eq. 2 , where $W$ is memductance of the memristor.

$$
\begin{gathered}
M\left(q_{m}(t)\right)=\frac{d \varphi_{m}\left(q_{m}(t)\right)}{d q_{m}(t)} \\
W\left(q_{m}(t)\right)=\frac{1}{M\left(q_{m}(t)\right)}
\end{gathered}
$$

As the fourth fundamental circuit element, memristor did not achieve much attention until 2008 when memristor was realized by Strukov et al from Hewlett-Packard lab[2]. HP memristor was composed by a sandwich structure with a doped $\mathrm{TiO}_{2}$ / undoped $\mathrm{TiO}_{2}$ junction which is also a low resistance/ high resistance junction. As been proved, when the charges flow through the device, the boundary between the two sides will move subsequently. The size of the doped region is a function of the charge flowing through the device and is responsible for the memristive effect as it changes the effective resistance of the device.

With the realization of memristor, its applications have attracted more and more attentions in different fields such as non-volatile memory, communication and neurography[3-5] etc. Especially in the field of neurography, circuits based on memory devices give a distinct construction of biological process. Pershin et al have shown that the behavior of unicellular organism Physarum subjected to periodic temperature and humidity variations can be analogical to the response of electronic circuit based on memory elements[6-7]. It is also suggested in Ref 6 that these behaviors are likely to occur in multicellular organisms, and the circuit model proposed can help researchers further investigate the origins of primitive intelligence and artificial intelligence. More importantly, as pointed by Ref 8, chaos is tightly related to the mem-circuit such as Hodgkin-Huxley axon circuit.

This paper is organized as follows: In section 2, we describe the models of Chua's circuit and memristor, and in section 3 we investigate the characteristics of canonical Chua's circuit and Chua's circuit based on memristor. Furtherly, the response characteristics of the circuits under the stimulating signals are researched. Section 4 is the conclusion of this paper. 


\section{Models and Circuits}

\subsection{Model of Chua's circuit}

As one of the most representative nonlinear circuit, Chua's circuit is consisted by the resistor, capacitor, inductor and nonlinear Chua's diode. The schematic of Chua's circuit is very simple, but its dynamic characteristic is very complex. In this circuit, the resistor is the component for energy consumption, while capacitor and inductor are for energy storage, and Chua's diode is for energy release. The schematic of the circuit is shown in Fig. 1(a).

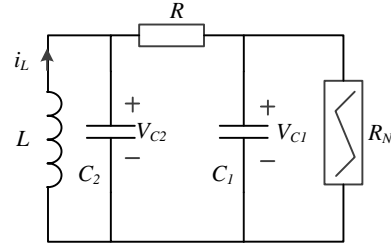

(a)

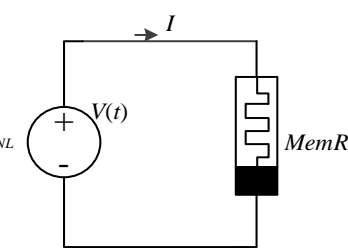

(b)

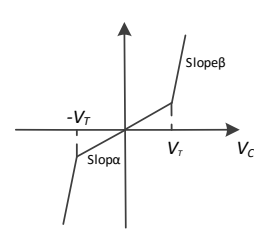

(c)

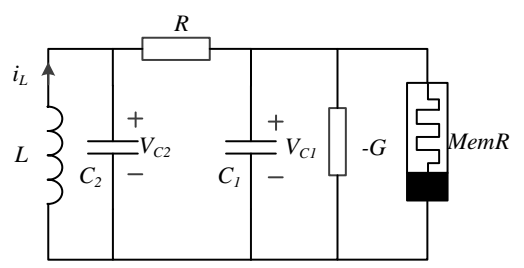

(d)

Fig.1(a) Chua's circuit; (b) Schematic of memristor;

(c) Characteristic of memristor; (d) Chua's circuit with memristor.

As shown in Fig. 1(a), $R_{N L}$ is Chua's diode and the conductance is defined by:

$$
g_{N L}(v)=g_{b} v+\frac{1}{2}\left(g_{a}-g_{b}\right)(|v+E|-|v-E|)
$$

where $v$ is the voltage drop on Chua's diode, $E$ is the turning voltage, and $g_{a}, g_{b}$ are the characteristic parameters. Through establishing and solving the state equations of Chua's circuit, we can get the change of voltages and currents with the time variation and also the phase diagram. The change of the voltages and currents in canonical Chua's circuit is very complex, and the waveform can be in a cyclical change form, chaos or tending to a steady state which is depended on the selection of parameters.

\subsection{Models of memristor}

As a fundamental memory element (see Fig. 1(b)), the typical features of memristor include: (1) the $v$ - $i$ curve is a pinched hysteresis and is periodic when a sinusoidal periodic signal is applied to the device; (2) the pinched hysteresis curve becomes weaker with increasing the input signal frequency; (3) the hysteresis loop will shrink into a straight line when the frequency increases to some large value.

A voltage controlled memristor is defined by:

$$
\begin{gathered}
I(t)=W\left(x, V_{M}, t\right) V_{M}(t) \\
\dot{x}=f\left(x, V_{M}, t\right)
\end{gathered}
$$

where $I(t)$ is the current flowing through the memristor, $V_{M}(t)$ is the input sinusoidal periodic voltage signal, $W$ is the memductance, $x$ is the state variable and the derivative of $x$ is equal to function $f\left(x, V_{M}, t\right)$ which is defined by Eq. 6 .

$$
f=\left(\beta V_{M}+0.5(\alpha-\beta)\left[\left|V_{M}+V_{T}\right|-\left|V_{M}-V_{T}\right|\right]\right) \times \theta\left(x-M_{1}\right) \theta\left(M_{2}-x\right)
$$

The characteristics of function $f$ is depicted in Fig. 1(c). In Eq. 6, $V_{M}$ is the voltage drop through memristor, $V_{T}$ is the threshold voltage, $\alpha$ is the slope when $\left|V_{M}\right|<V_{T}, \beta$ is the slope when $\left|V_{M}\right|>V_{T}, \theta$ is the step function, $M_{1}$ is minimum value of memristance while $M_{2}$ is the maximum value.

\subsection{Model of Chua's circuit with memristor}

As shown in Fig. 1(d), Chua's diode has been replaced by a negative conductor and a memristor. The state equations of this circuit are as follows:

$$
\begin{gathered}
C_{1} \frac{d v_{1}}{d t}=\frac{v_{2}-v_{1}}{R}+v_{1} G-f\left(v_{1}\right) \\
C_{2} \frac{d v_{2}}{d t}=\frac{v_{1}-v_{2}}{R}+i_{L} \\
L \frac{d i_{L}}{d t}=-v_{2}-r \cdot i_{L}
\end{gathered}
$$

Supposing $x=v_{1}, y=v_{2}, z=i_{L} R, d t=C_{2} R d \tau, t=C_{2} R d \tau, a=C_{2} / C_{1}, b=R_{2} \cdot C_{2} / L, \varepsilon=R_{2} \cdot C_{2} / L, p=1-R G$ and $g(x)=R f(x)$, the equations above read: 


$$
\begin{gathered}
\frac{d x}{d \tau}=a[y-p x-g(x)] \\
\frac{d y}{d \tau}=x-y+z \\
\frac{d z}{d \tau}=-b y-\varepsilon z
\end{gathered}
$$

By solving these equations, we can achieve the different states of this circuit.

\section{Results and Discussion}

\subsection{Characteristics of Chua's circuit and responses to stimulating signals}

Characteristics of Chua's circuit are closely related to the selection of circuit parameters, and careful setting of the parameters can make the circuit stay in different states. As shown in Figure 2, phase trajectory diagrams of Chua's circuit have been achieved in different states, e.g. double scroll state (see Fig. 2(a)), single scroll state (see Fig. 2(b)), multi-periodic state (see Fig. 2(c)) and single-periodic state (see Fig. 2(d)).

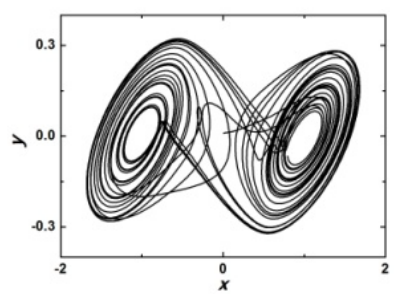

(a)

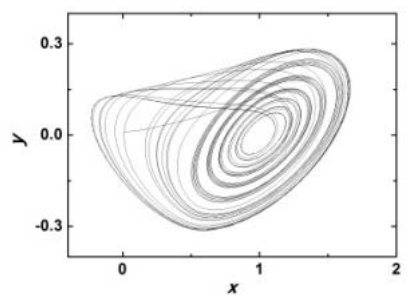

(b)

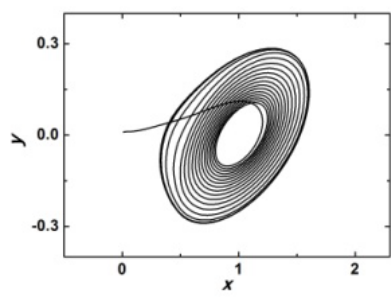

(c)

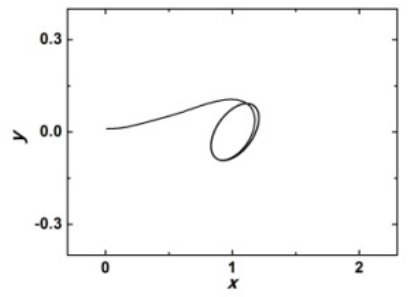

(d)

Fig.2(a) Double scroll state; (b) Single scroll state; (c) Multi-periodic state; (d) Single-periodic state

Based on the results we have gotten above, we continue to investigate the characteristics of the response to the external stimulating signals. Single-periodic state of the circuit is chosen as initial state and a pulse signal of current is added to the terminals of Chua's diode. The pulse signal we added is a square wave with the period equal to that of the initial single-periodic state. Then, the amplitude $A$ of the square wave is varied and the phase trajectory diagrams have been achieved.

As shown in Fig. 3, external stimulating signals could change the circuit state significantly, and the transition is dependent on the parameter which is the amplitude $A$ we choose here. When the amplitude $A$ equals to $0.5,6$ periods of the pulse at least are needed to promote the transition, while 4 , 3 and 2 periods at least are needed when $A$ increases to 0.6, 0.8 and 1. It is suggested that, it is easier to make the transition when the amplitude is larger. Besides, the final stable states of the circuit have differences to each other which are also related to the amplitude of the external stimulating signals.

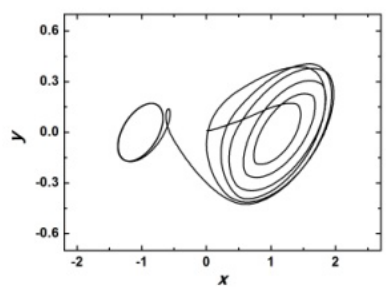

(a)

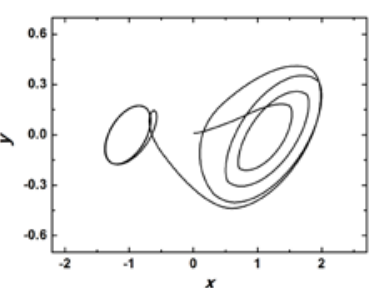

(b)

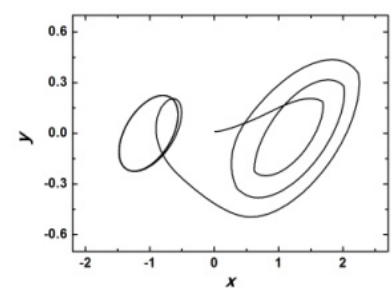

(c)

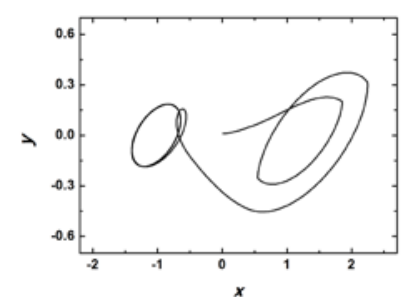

(d)

Fig. 3(a) $A=0.5$; (b) $A=0.6$; (c) $A=0.8 ; \quad$ (d) $A=1$.

\subsection{Characteristics of Chua' circuit with memristor and responses to stimulating signals}

As a typical non-linear element, memristor has been used to generate oscillating signals and investigate the phenomenon chaos. Fig. 4 is the characteristics of Chua's circuit with memristor and under the stimulating signals. By selecting different parameters, the circuit can be in various states. Fig. 4(a) is the phase diagram of circuit without external signal and the circuit is in the state of stable focus. 
In order to investigate the state transitions of the circuit under stimulating signals, we choose the state of stable focus as initial, and the stimulating signals are periodic pulse signal, square wave signal with 5 periods and sinusoidal periodic signal respectively, as shown in Fig. 4(b), Fig. 4(c) and Fig. 4(d). As we can see, the initial state of stable focus has been changed when the external stimulating signals are applied, and the complex chaotic characteristics are presented.

Based on the results above, it is suggested that, the stimulating signals can change the circuit states significantly. However, the phenomenon of chaos is so complex, and the transitions are dependent on the parameters, especially closely related to the range of definite parameter. Up to now, we have not made this phenomenon clear, and proposed the relevant mechanism. Even though, this process has significant meaning on memory-effect and related neurography.
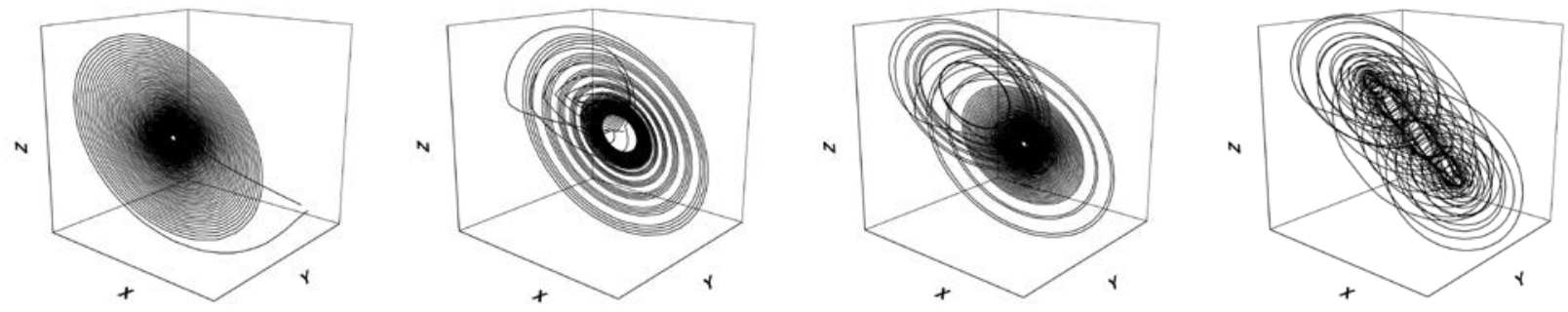

Fig. 4(a) Without external stimulating signal; (b) With periodic pulse signal;

(c) With 5 periods square wave signal; (d) With sinusoidal periodic signal.

\section{Conclusions}

In conclusion, by investigating the characteristics of canonical Chua's circuit and Chua's circuit based on memristor, it is suggested that the external stimulating signals could change the initial states of the circuits significantly. Simultaneously, the transition of the states is dependent on the section of relevant parameters. It may thus find applications in communication and neurography. Moreover, the results can help researchers further investigate the origins of primitive intelligence and artificial intelligence.

\section{References}

[1]. Chua L O. Memristor - the missing circuit element[J]. IEEE Transactions on Circuit Theory, 1971, 18(5): 507-519.

[2]. Strukov D B, Snider G S, Stewart D R, Stanley Williams R. The missing memristor found[J]. Nature, 2008, 453: 80-83.

[3]. Chua L O. Resistance switching memories are memristors[J]. Applied Physics A, 2011, 102: 765-783.

[4]. Kvatinsky S, Friedman E G, Kolodny A, Weiser U C. TEAM: ThrEshold Adaptive Memristor Model[J]. IEEE Transactions on Circuits and Systems I: Regular Papers, 2013, 60(1): 211-221.

[5]. Di Ventra M, Pershin Y V, Chua L O. Circuit Elements With Memory: Memristors, Memcapacitors, and Meminductors[J]. Proceedings of the IEEE, 2009,97(10):1717-1724.

[6]. Pershin Y V, Fontaine S L, Di Ventra M, Memristive model of amoeba learning[J]. Physical Review E, 2009, 80: 021926(1)- 021926(6).

[7]. Pershin Y V, Di Ventra M, Experimental demonstration of associative memory with memristive neural networks[J]. Neural Networks, 2010, 23(7): 881-886.

[8]. Chua L O, Memristor, Hodgkin-Huxley, and Edge of Chaos[J], Nanotechnology, 2013, 24: 383001(1)-383001(14) 